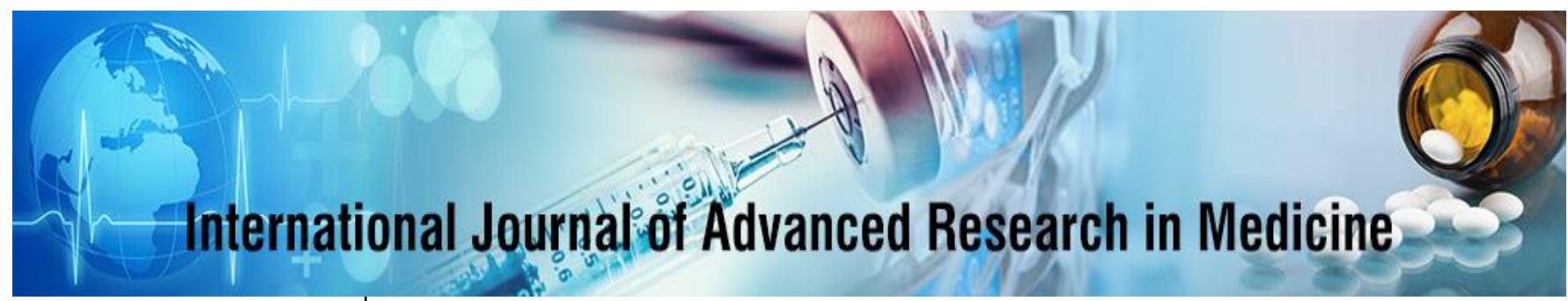

E-ISSN: 2706-9575

P-ISSN: 2706-9567

IJARM 2021; 3(1): 456-458

Received: 10-05-2020

Accepted: 14-06-2020

\section{Dimitrios Goulios}

Department of Physiotherapy,

Faculty of Health Sciences

International Hellenic

University Alexander Campus,

Sindos, Thessaloniki, Greece

Dimitrios Lytras

Department of Physiotherapy,

Faculty of Health Sciences

International Hellenic

University Alexander Campus,

Sindos, Thessaloniki, Greece

\section{Paris Iakovidis}

Department of Physiotherapy,

Faculty of Health Sciences

International Hellenic

University Alexander Campus,

Sindos, Thessaloniki, Greece

\section{Anastasios Kottaras}

Department of Physiotherapy,

Faculty of Health Sciences

International Hellenic

University Alexander Campus,

Sindos, Thessaloniki, Greece

Konstantinos Moutaftsis

Department of Physiotherapy, Faculty of Health Sciences

International Hellenic

University Alexander Campus,

Sindos, Thessaloniki, Greece

Georgios Leptourgos

Department of Physiotherapy,

Faculty of Health Sciences

International Hellenic

University Alexander Campus,

Sindos, Thessaloniki, Greece

Corresponding Author: Dimitrios Goulios

Department of Physiotherapy,

Faculty of Health Sciences

International Hellenic

University Alexander Campus,

Sindos, Thessaloniki, Greece

\section{The effect of electrotherapy on traumatic lesions of the sciatic nerve: A review of evidenced-based physiotherapy practice}

\author{
Dimitrios Goulios, Dimitrios Lytras, Paris Iakovidis, Anastasios \\ Kottaras, Konstantinos Moutaftsis and Georgios Leptourgos
}

DOI: https://doi.org/10.22271/27069567.2021.v3.i1h.180

\begin{abstract}
Damage to the sciatic nerve is rare but causes pain that is not easily relieved, thus often necessitating hospitalization. The most common causes of injury are hip surgery, local injections, amputation and hip fracture. This review aims to describe research data on the efficacy of electrotherapy applications in cases of sciatic nerve injury. The Google Scholar and PubMed databases were searched in English with the following keywords: electrotherapy, sciatic injury, physiotherapy, efficacy. Both clinical trials and systematic reviews were included in the review. This review included seven clinical trials and two systematic reviews on the efficacy of various electrotherapy methods. The results of the review showed that electrotherapy can help improve the pain resulting from damage to the sciatic nerve. According to the results of this review, the application of electro acupuncture (EA), Percutaneous Electrical Nerve Stimulation (PENS), Transcutaneous Electrical Nerve Stimulation (TENS) and Medium-Frequency Electrotherapy (MFE) is considered to have a positive effect, although the results of the studies in many cases appear ambiguous. By comparison, EA is considered more efficient. PENS was significantly more effective than TENS in improving physical activity, pain and sleep quality, and postoperative function. New methods, such as the application of Peripheral Nerve Stimulation (PNS) electrodes with the use of ultrasound, need to be better evaluated in the coming years, but they look very promising.
\end{abstract}

Keywords: Electrotherapy, sciatic injury, physiotherapy efficacy

\section{Introduction}

The sciatic nerve is the largest nerve in the human body. It is formed from the L4-L5 and S1$\mathrm{S} 3$ roots of the sacral plexus by the union of the tibial and peroneal nerves. The sciatic nerve passes beneath the piriformis muscle and from there exits to the back of the leg. The sciatic nerve and its branches innervate a large number of muscles of the lower limb and supply sensation to most of the foot ${ }^{[1]}$. Patients often use the term sciatica to describe pain radiating down the leg, but in fact a sciatic nerve injury is rare. The most common cause of actual sciatic nerve injury is hip surgery, injection, amputation and hip fracture. External injuries (e.g., gunshot wounds) may also affect the sciatic nerve. Other types of conditions that affect the sciatic nerve include even nerve compression that can occur in the pelvis from neoplasms or retroperitoneal bleeding. When the sciatic nerve is damaged, weakness can occur in the extensor muscles of the hip and the dorsal extensors of the leg ${ }^{[2]}$. Various environmental and genetic factors that are considered to influence the development of hip damage and pain have been studied, including gender, posture, age, genetic factors, occupation, and environmental variables ${ }^{[3]}$

Recent epidemiological data from the National Database of the United States of America on patients with sciatic nerve injury admitted to US hospitals from 2001 to 2013 indicate that these patients accounted for $16.6 \%$ of admissions with a mean age of $41.6 \pm 0.1$ years. In addition, $51 \%$ of hospitalized patients are eventually discharged, while $28.2 \%$ are referred for further treatment to a specialized hospital. The main cause of injury was surgical complications amounting to $68.8 \%$, whereas $13.6 \%$ of these lesions were due to a lower limb fracture ${ }^{[4]}$.

In terms of treatment, there are several options for treating peripheral nerve injuries, as well as many new strategies for physiotherapy rehabilitation protocols that have positive effects 
after an injury. Physiotherapy aims to compensate for the dysfunctions while improving the sensory disorders and contributing to the faster healing of the nerve, thus being an essential part of the treatment after peripheral nerve injuries. Such approaches are kinesiotherapy, electrotherapy, therapeutic laser and others ${ }^{[5,6]}$.

This literature review aims to describe research data on the use of electrotherapy in cases of sciatic nerve damage as well as to present the types of electrotherapy treatments applied, their different parameters and their effects on the symptoms of the patients.

\section{Method}

The Google Scholar and PubMed were searched in English with the following keywords: electrotherapy, sciatic injury, physiotherapy efficacy. The review included both clinical trials and systematic reviews.

\section{Results}

In total, nine articles were included in this review. Below are their main findings.

\section{Literature review}

Numerous reviews on the physiotherapy approach for peripheral nerve damage have shown that electrotherapy can contribute significantly to the clinical picture of these patients ${ }^{[7,8]}$. The methods of electrotherapy in the treatment of peripheral nerve lesions vary depending on the desired result, the degree and stage of the injury. According to Suszyński et al. ${ }^{[5]}$, the application of Transcutaneous Electrical Nerve Stimulation (TENS) with frequencies of $90-130 \mathrm{~Hz}$ offers a satisfactory analgesic effect for the treatment of pain in the acute phase also report that there are other methods with good results such as Percutaneous Electrical Nerve Stimulation (PENS). In PENS electrical stimulation passes from the skin to the tissue with needlelike electrodes. However, as the authors note, there is an insufficient number of studies to allow critical conclusions to be drawn.

In the clinical trial of Ghoname et al. ${ }^{[9]}$, the application of two different electrotherapy methods (PENS and TENS) was compared in the management of sciatica-related pain in 64 patients with intervertebral disc herniation. The participants were divided into three groups; two intervention (TENS and PENS) and a control group. The stimulation frequency for both protocols was $4 \mathrm{~Hz}$. Each treatment was administered for a period of 30 minutes three times a week for three weeks, with one break week between each treatment. PENS (42\%) and TENS (23\%) were more effective than the control group $(8 \%)$ in reducing pain. Analgesic medication use was also significantly reduced compared to pre-PENS $(p<.01)$ and pre-TENS $(p<.05)$ values. PENS was significantly more effective than TENS and placebo PENS in improving physical activity, sleep quality and post-operative functioning.

A recently published technique by Singh ${ }^{[10]}$ describes percutaneous placement of Peripheral Nerve Stimulation (PNS) electrodes, using ultrasound to locate the sciatic nerve and design the needle trajectory. The technique allows parallel or vertical orientation of an electrode to the target nerve. The needle path is determined and a catheter is inserted and directed along the programmed trajectory at a distance of $5-15 \mathrm{~mm}$ from the target nerve. Electrical stimulation is then used to determine the ideal position to inhibit pain transmission in the affected area. Once the optimal stimulation point has been determined, the sensor is replaced with the electrode. The authors believe that this method may be more effective in treating pain from the sciatic nerve, but state that more research is needed to determine its efficacy.

In another clinical trial by Okonkwo et al. ${ }^{[11]}$, the effects of TENS on the management of sciatic nerve pain due to injection injury were evaluated. A total of 72 patients participated in a non-randomized controlled clinical trial (40 participants in the intervention group and 32 in the control group). TENS and placebo TENS were applied to the intervention and control group respectively, three times a week and one hour per session for the 10 weeks of the study. The results showed that the level of pain among the participants in the treatment group at the end of the intervention was significantly lower than that of those in the control group $(\mathrm{F}=16.26, p=.01)$.

The study by Zhang et al ${ }^{[12]}$ investigated the short- and long-term effects of electroacupuncture (EA) compared to Medium-Frequency Electrotherapy (MFE) in chronic discogenic sciatica. A total of 100 participants were randomized into two groups to receive EA $(n=50)$ or MFE $(n=50)$ for four weeks. Both groups were monitored for 28 weeks. The mean changes in the mean leg pain scores by the fourth week were $2.30(1.86-2.57)$ for the EA group and $1.06(0.62-1.51)$ for the MFE group. The difference was statistically significant $(p<.001)$. At week 28 , mean leg pain scores decreased by $2.12(1.70-2.53)$ in the EA group and $0.36(-0.05-0.78)$ in the MFE group, compared to baseline. EA presented greater short- and long-term benefits for chronic discogenic sciatica than MFE.

An older study ${ }^{[13]}$ also used EA for chronic sciatica, in a single-blind clinical trial compared to physiotherapy and placebo pharmacotherapy. In total, 119 people (59 men, 65 women) were selected and classified into three treatment groups consisting of: EA, physiotherapy and placebo. The rate of pain reduction in the EA, physiotherapy and placebo groups was $62.1 \% \pm 18.6 \%, 52.5 \% \pm 17.5 \%$ and $17.5 \% \pm$ $12.7 \%$ respectively $(p<.05)$. Satisfaction in the EA group was significantly higher than the other two groups $(p<.01)$. The rate of reduction of complications in the EA, physiotherapy and placebo groups was $89.3 \%, 51.8 \%$ and $31.9 \%$, respectively $(p<.05)$. EA was more effective than physiotherapy in managing pain $(p<.05)$.

Immediate sciatic nerve stimulation has the benefit of reducing lower limb pain. The nerve fibers in the leg are difficult to stimulate with electrodes placed in the spine because they are deep. A peripheral approach to the sciatic nerve can overcome these limitations by transcutaneous nerve stimulation via electrode implantation ${ }^{[14]}$.

The study conducted by Gilmore et al. ${ }^{[15]}$ investigated pain from sciatic nerve injury after amputation and whether percutaneous peripheral nerve stimulation (PNS) can help reduce it. The 28 participants underwent transcutaneous PNS electrode implantation and were randomized to receive PNS or placebo for four weeks. The control group then received PNS for an additional four weeks. A significantly higher proportion of people receiving PNS $(n=7 / 12,58 \%$, $p=.037$ ) showed at least $50 \%$ reductions in mean pain after treatment compared with people receiving placebo $(\mathrm{n}=$ $2 / 14,14 \%$ ). A significantly higher proportion of people with PNS also reported at least $50 \%$ reduction in pain $(n=8 / 12$, $67 \%, p=.014)$ after eight weeks of treatment compared 
with placebo $(n=2 / 14,14 \%)$. Four of the five PNS individuals who completed 12 months of follow-up reported at least $50 \%$ pain relief.

Mobbs et al. ${ }^{[16]}$ evaluated an implanted peripheral nerve stimulator in patients with peripheral nerve pain (such as sciatica). The patient sample included 38 patients (41 nerve stimulators). Participants were divided into four groups based on the etiology of their injuries: blunt or sharp nerve injury (14/38), iatrogenic surgical injuries (9/38), involuntary nerve injection (9/38) and post-operative intervention (8/38). Stimulation was attempted in 45 patients, but the trial was not successful in four. Preoperative pain relief was considered good (> 50\%) by $23 / 38$ patients $(61 \%)$. A total of 15 patients $(39 \%)$ reported moderate or poor results, while six patients $(15 \%)$ needed the stimulator to be removed due to infection or reduced pain control after an initial good result. A statistically significant reduction in the reported level of pain was found post-operatively $(p<.05)$. Lastly, $18 / 38$ patients $(47 \%)$ reported a significant improvement in their activity levels after implantation.

\section{Discussion and Conclusions}

From the results of the review, it is made clear that electrotherapy can help improve the pain from hip injury. Due to the lack of studies in humans investigating nerve reconstruction, only the presence of pain in patients could be assessed. EA, PENS, TENS and MFE are considered efficient by studies with varying results. In comparison, EA should be considered more efficient [12, 13]. PENS was significantly more effective than TENS in improving physical activity, pain, sleep quality and post-operative functioning ${ }^{[9]}$.

New methods (such as the placement of PNS electrodes, using ultrasound to locate the sciatic nerve and design the needle trajectory) need to be better evaluated in the coming years, but they look very promising ${ }^{[10]}$. A study evaluating this new method reports a higher proportion of people with PNS reporting at least $50 \%$ reduction in pain after eight weeks of treatment compared with people treated with placebo ${ }^{[15]}$.

Our understanding of the pathophysiology of sciatic nerve damage and how to treat it has improved significantly in recent years. Some interesting methods have been developed, but have not yet been sufficiently explored. Currently available electrotherapy treatments can reduce pain without modifying the long-term clinical course of hip injury.

\section{References}

1. Ropper AH, Zafonte RD. Sciatica. New England Journal of Medicine 2015;372(13):1240-8.

2. Brust JCM. Current diagnosis \& treatment neurology: McGraw Hill Professional 2018.

3. Kumar M, Garg G, Singh LR, Singh T, Tyagi LK. Epidemiology, pathophysiology and symptomatic treatment of sciatica: a review. Int. J Pharm Biol Sci Arch 2011;2(4):1050-61.

4. Foster CH, Karsy M, Jensen MR, Guan J, Eli I, Mahan MA. Trends and Cost-Analysis of Lower Extremity Nerve Injury Using the National Inpatient Sample. Neurosurgery 2018;85(2):250-6.

5. Suszyński K, Marcol W, Górka D. Physiotherapeutic techniques used in the management of patients with peripheral nerve injuries. Neural Regen Res 2015;10(11):1770-2.

6. Ward AR. 10.11 - Electro-Muscle Stimulation Therapy. In: Brahme A, editor. Comprehensive Biomedical Physics. Oxford: Elsevier, 2014, pp. 231-53.

7. Lytras D, Sykaras E, Semaltianou E, Myrogiannis IS. Physiotherapy Treatment in Nontraumatic Individual Nerve Entrapment Syndromes of the Brachial Plexus in Athletes: A Clinical Presentation Related to Injury from Sports Activities and Physiotherapy Management Clinical Guidelines. Critical Reviews ${ }^{\mathrm{TM}}$ in Physical and Rehabilitation Medicine, 2020, 32(4).

8. Valat JP, Genevay S, Marty M, Rozenberg S, Koes B. Sciatica. Best Practice \& Research Clinical Rheumatology 2010;24(2):241-52.

9. Ghoname EA, White PF, Ahmed HE, Hamza MA, Craig WF, Noe CE. Percutaneous electrical nerve stimulation: an alternative to TENS in the management of sciatica. Pain 1999;83(2):193-9.

10. Singh A, Gargya A, Lin T, Gulati A. Sciatic, Femoral, and Lateral Femoral Cutaneous Nerve UltrasoundGuided Percutaneous Peripheral Nerve Stimulation. Pain Medicine 2020;21(1):S47-S50.

11. Okonkwo UP, Ibeneme SC, Ihegihu EY, Egwuonwu $\mathrm{AV}$, Ezema IC, Maruf AF et al. Effects of transcutaneous electrical nerve stimulation in the Management of Post-Injection Sciatic Pain in a nonrandomized controlled clinical trial in Nnewi, Nigeria. BMC complementary and alternative medicine 2018;18(1):1-11.

12. Zhang X, Wang Y, Wang Z, Wang C, Ding W, Liu Z. A randomized clinical trial comparing the effectiveness of electro acupuncture versus medium-frequency electrotherapy for discogenic sciatica. Evidence-Based Complementary and Alternative Medicine. 2017;2017.

13. Hollisaz MT. Use of electroacupuncture for treatment of chronic sciatic pain. Internet $\mathbf{J}$ Pain Symptom Control Palliative Care 2006;5(1):1-4.

14. Siddalingaiah V, Goroszeniuk T, Palmisani S. Permanent percutaneous sciatic nerve stimulation for treatment of severe neuropathic pain. Proceedings of the 13th World Congress on Pain; Montreal, Canada 2010.

15. Gilmore C, Ilfeld B, Rosenow J, Li S, Desai M, Hunter $\mathrm{C}$, et al. Percutaneous peripheral nerve stimulation for the treatment of chronic neuropathic postamputation pain: a multicenter, randomized, placebo-controlled trial. Regional Anesthesia \&amp; amp; Pain Medicine. 2019:rapm-2018-100109.

16. Mobbs RJ, Nair S, Blum P. Peripheral nerve stimulation for the treatment of chronic pain. Journal of clinical neuroscience 2007;14(3):216-21. 